\title{
Los idiomas mesoamericanos de Nicaragua en el contexto centroamericano Informe de una investigación
}

\author{
Danilo Salamanca
}

El presente documento es un informe de una investigación sobre los idiomas mesoamericanos que se hablaron en Nicaragua, llevada a cabo con el patrocinio de IbisDinamarca en dos períodos: de octubre y noviembre del 2007 y de junio a diciembre del 2009. La investigación se realizó como parte del apoyo brindado por esta organización danesa a las reivindicaciones territoriales y culturales de las organizaciones indígenas del Pacífico, Centro y Norte de Nicaragua ${ }^{1}$.

En efecto, los movimientos reivindicativos indígenas tanto en Nicaragua como en el resto del continente, como parte de sus luchas buscan conocer mejor su historia y sus raíces culturales que en gran parte han sido borradas por siglos de opresión ${ }^{2}$. La recuperación de información sobre las lenguas ancestrales, cuando éstas han dejado de hablarse en las comunidades indígenas supervivientes, es naturalmente una parte esencial de ese esfuerzo ${ }^{3}$.

Aparte de inventariar y recopilar las fuentes de información sobre las lenguas y las culturas indígenas mesoamericanas de Nicaragua, nuestro trabajo investigativo comprendió visitas a Costa Rica, Honduras y el Salvador, para conocer mejor las investigaciones y trabajos de revitalización cultural y lingüística existentes en esos países, especialmente aquellos que conciernen a pueblos mesoamericanos afines a los de Nicaragua.

El presente informe resume los resultados del trabajo investigativo, los contactos que realizamos con académicos y líderes culturales indígenas en los países vecinos, algunas iniciativas de colaboración que surgieron de esos contactos y varias maneras en que el trabajo iniciado podría ser continuado y expandido.

\section{El objeto de nuestra investigación.}

Basado en las crónicas, documentos históricos e investigaciones que se han realizado sobre el período con el auxilio de diversas disciplinas, en particular la arqueología y la lingüística, existe cierto consenso en el ámbito académico ${ }^{4}$ sobre nuestras raíces lingüísticas indígenas, el cual aparece también reflejado en los manuales escolares o universitarios que tratan sobre nuestra historia antigua ${ }^{5}$. Lo repasaremos brevemente para definir más precisamente el objeto de nuestra investigación.

Las fuentes académicas y los textos de historia establecen la existencia de dos tipos de indígenas en el territorio de la actual Nicaragua a la llegada de los españoles. Los llamaremos respectivamente macrochibchas y mesoamericanos.

\section{Macrochibchas}

Por una parte, los miskitos, mayangnas, ulwas y ramas de las actuales regiones autónomas RAAN y RAAS ${ }^{6}$ son descendientes de los grupos lingüísticos de filiación chibchence -algunos de los cuales se extinguieron- que

\footnotetext{
1 Para un inventario de los movimientos indígenas contemporáneos en el Pacifico y Centro de Nicaragua ver Olguín Martínez (2006)

2 La información obtenida sobre la historia y cultura ancestrales puede entonces ser integrada en los currículos escolares de estos pueblos. Un ejemplo de este tipo de esfuerzo relativo a uno de los pueblos de la Coordinadora Chorotega es Rizo (2008).

${ }_{3}$ Este vivo interés, al igual que sugerencias sobre la manera de utilizar y ampliar, a partir de tradiciones y conocimientos conservados en las comunidades, los resultados de la investigación que estábamos realizando sobre los idiomas, nos fueron manifestados por dirigentes de la coordinadora chorotega en la reunión que sostuvimos con ellos el 16 de septiembre del 2009.

4 Por ejemplo, Lehmann 1910 y 1920, Campbell 1979, Salamanca 1993, (1994) y Constenla 1994 entre otros.

Ver por ejemplo las “Historia de Nicaragua” de Romero Vargas (2003) y la de Kinloch Tijerino (2006)

6 Los garífunas fueron deportados en 1797 por las autoridades inglesas a partir de la isla caribeña de Saint Vincent a la Isla de Roatán en Honduras; de ahi, éstos se extendieron por las costas de Centroamérica. Para una historia cultural de los garífunas ver Coehlo (1995).
} 


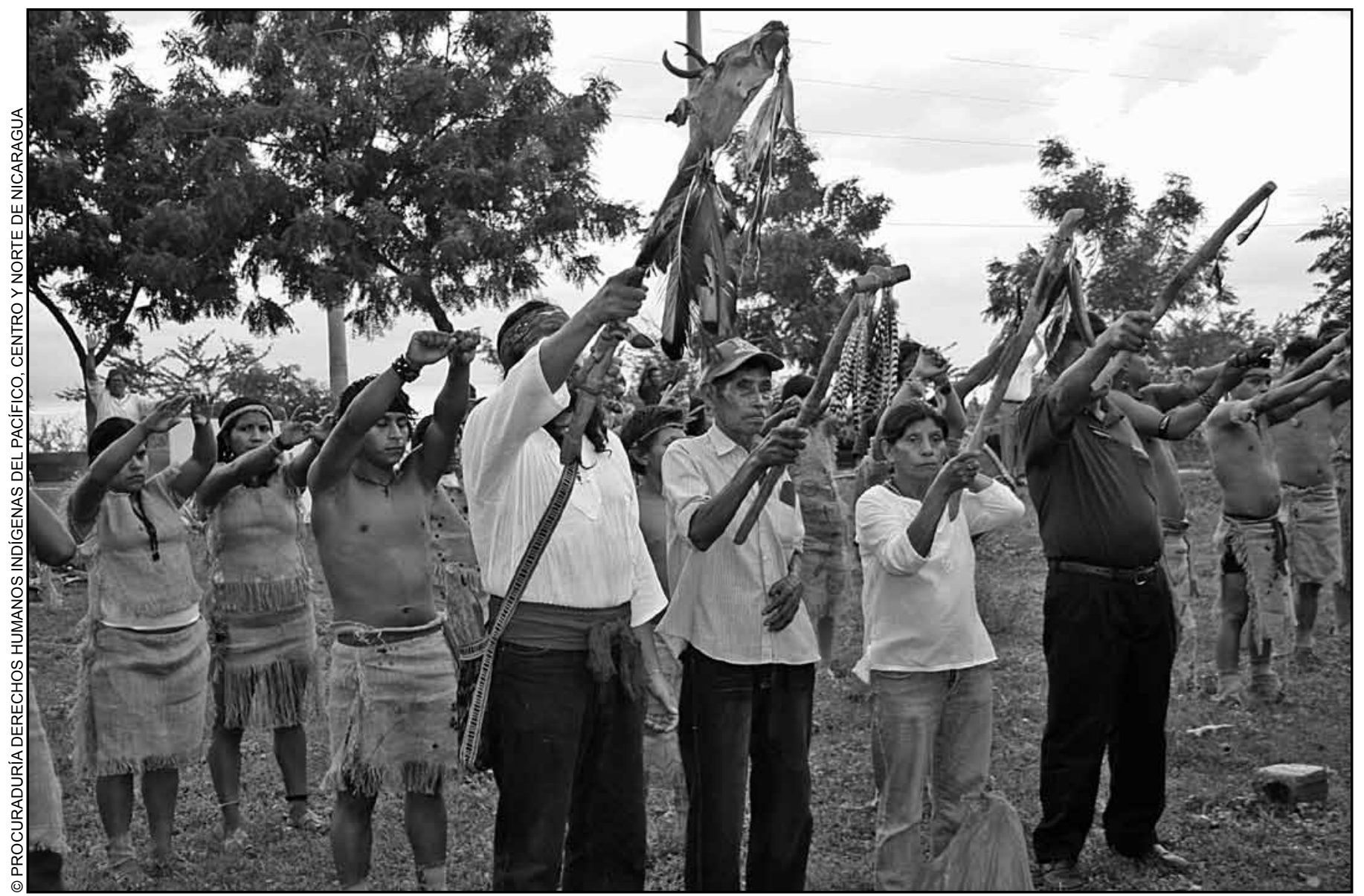

Indígenas del Pacífico, Centro y Norte de Nicaragua durante una ceremonia, 2010.

ya en esa época poblaban esa vertiente del país al igual que gran parte del centro de Nicaragua. Los matagalpas, que vivían hacia el centro-norte y fueron integrados a la sociedad colonial española, son por sus lenguas y culturas originales miembros de esa gran familia.

Los matagalpas junto con los miskitos, mayangnas y ulwas conforman una familia de lenguas conocidas con el nombre de misumalpa, que se distingue de la familia chibcha propiamente y tiene un vínculo histórico más cercano con la familia de idiomas lencas que se hablaron en Honduras y el Salvador. Los ramas, por su parte, junto con los payas o pech de Honduras están más estrechamente emparentados con los miembros de la familia chibcha, cuyo territorio ancestral se extiende por Costa Rica, Panamá, Colombia y todavía más allá en el continente?

La gran familia Macro-chibcha está formada por la familia misumalpa y los idiomas lencas por un lado y la diversas ramas de la familia chibcha propiamente por el otro.

En el archipiélago de Solentiname y en el río san Juan había también otros grupos indígenas de estirpe chibchence con los cuales las crónicas y diversas investigaciones asocian el nombre corobici. Su idioma habría sido próximo al guatuso, que todavía se habla en Costa Rica y que también es cercano al rama ${ }^{8}$.

\footnotetext{
Para una discusión amplia sobre la familia chibcha propiamente ver los trabajos de los lingüistas costarricenses Quesada (2007) y Constenla Umaña (1991); para la relación entre los idiomas lencas y los idiomas misumalpas ver Constenla Umaña (2002). Los trabajos citados de Constenla, al igual que Constenla Umaña (2005) contienen apreciaciones acerca de las bifurcaciones en el tiempo de los idiomas y familias de idiomas.

8 Lehmann (1910), pg 707-708, señala que a finales del siglo XVIII indígenas de Solentiname fueron llevados como interpretes por religiosos enviados en misión hacia los guatusos y deduce razonablemente que esto implica que se sabía que algún grado de comunicación era posible entre ellos.
} 


\section{Mesoamericanos}

Por otra parte, en las tierras fértiles de la vertiente del Pacifico y en las márgenes de los lagos vivían indígenas agricultores que presentaban afinidades y filiaciones culturales y lingüísticas con grupos de indígenas situados hacia el norte en el continente, hasta México y más allá. De estas culturas y lenguas, caracterizadas en la literatura académica como "mesoamericanas", que incluyen a los mayas y aztecas más al norte, las crónicas y la historiografía tradicional han identificado a tres de los cuales ha quedado alguna evidencia lingüística: los nicarao, los chorotegas y los subtiava ${ }^{10}$.

En Nicaragua ninguno de esos tres idiomas era ya utilizado corrientemente para comienzos del siglo XX cuando el investigador Walter Lehmann hizo una minuciosa visita al país, para colectar información directa sobre los idiomas que entonces se hablaban todavía. Sin embargo, Lehmann pudo todavía recoger preciosos datos sobre el idioma subtiava de una anciana hablante de 84 años, Victoria Carrillo, que en la opinión de Lehmann tenía un conocimiento sólido del idioma, así como de dos otros semi-hablantes en el pueblo del mismo nombre, un suburbio de la ciudad de León.

Además de en la franja del Pacifico de Nicaragua, el chorotega también se habló en la región de Guanacaste que actualmente es parte de Costa Rica, al igual que en la región de Choluteca en Honduras.

El nicaro por su parte, era una variante o dialecto del nahuatl, el idioma de los aztecas del cual sobreviven en México varios dialectos. Este idioma estaba presente también en otras partes de Centroamérica, y en particular al occidente del actual territorio de El Salvador donde todavía hay hablantes.

Como el idioma de los corobici que se habló al sur del gran lago era miembro de la familia de idiomas chibcha y el matagalpa conforma con el miskito y los idiomas sumos la familia misumalpa (el nombre es una abreviación de miskito sumo y matagalpa), ambas familias implantadas en la vertiente atlántica del país, y sobre los cuales se han llevado a cabo numerosas investigaciones en los últimos años ${ }^{11}$, el objeto del presente estudio sobre los idiomas mesoamericanos de la vertiente del Pacifico y centro de Nicaragua son los idiomas: Nahuatl, Chorotega y Subtiava.

El proyecto de investigación sobre estos idiomas tenía dos componentes principales.

I) ) Por un lado constituir un fondo bibliográfico. Tanto en Nicaragua como en Costa Rica, el Salvador y Honduras tratamos de adquirir las obras y trabajos de naturaleza académica sobre las lenguas, así como también documentos históricos y otros materiales que nos informaran sobre la situación actual de los grupos étnicos sobre los cuales queremos saber más en Nicaragua y en los países vecinos. Estos materiales fueron reunidos con la intención de ir constituyendo un fondo bibliográfico disponible a agrupaciones indígenas para diferentes fines: investigativos, pedagógicos, etcétera.

II) Por otro lado realizar visitas a los diferentes países vecinos para entrevistarnos con lingüistas y otros especialistas de la cuestión indígena, así como con líderes y activistas culturales indígenas en cada país: Costa Rica (en el 2007), Honduras y El Salvador (en la segunda mitad el 2009).

El informe está compuesto de las siguientes secciones: En la sección 2 vamos a presentar un resumen de lo que se ha podido establecer sobre las lenguas mesoamericanas que se hablaron en Nicaragua a partir de la literatura especializada. Cada vez que nos fue posible tratamos de incluir en el fondo bibliográfico los trabajos ahí citados. En la sección 3 haremos un recuento y resumen de los resultados de las visitas realizadas a cada uno de los países vecinos. Los contactos académicos que establecimos durante ellas han sido consolidados a través de la creación de la Asociación Centroamericana de Lingüística (ACALING), que tiene dentro de sus principales objetivos la colaboración internacional para estudiar y promover las lenguas indígenas de la región. La sección 4 presenta algunas conclusiones y comentarios sobre las perspectivas para continuar el trabajo de investigación que aquí vamos a presentar, en el contexto de las luchas por sus derechos y reconocimiento de los pueblos indígenas concernidos en cada uno de los países visitados y particularmente en Nicaragua.

\footnotetext{
9 Para una discusión sobre el término “mesoamericano” en ese sentido ver Hasemann (1991).

10 En las crónicas aparecen también mencionados los nombres "tacacho” y "potón” cuya identidad lingüistica u otra no ha podido ser aclarada satisfactoriamente, su implantación en todo caso habría sido muy leve.

1 Para un recuento de los principales trabajos descriptivos realizados sobre los idiomas macrochibchas de Nicaragua ver Salamanca (2007).
} 


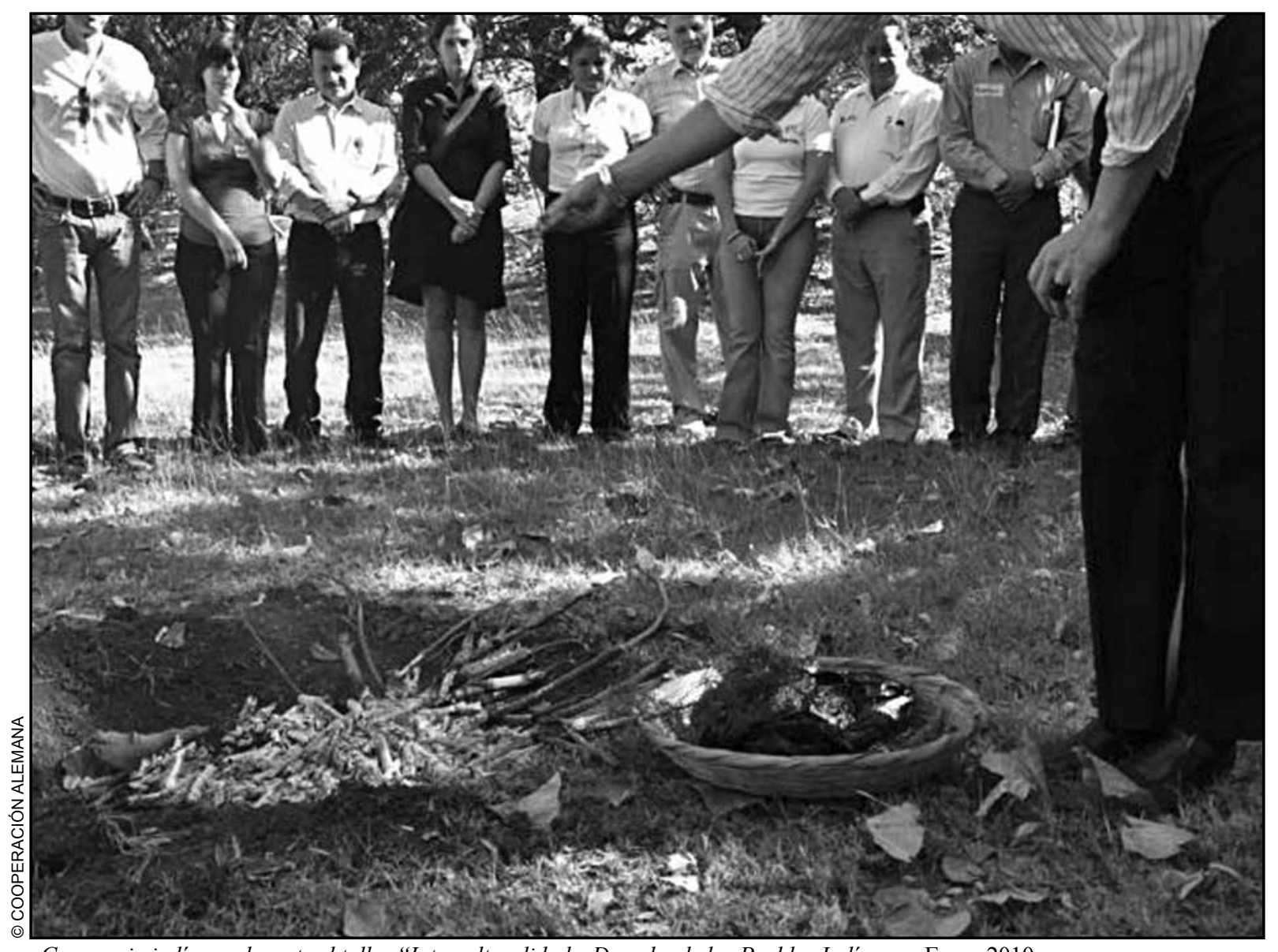

Ceremonia indigena durante el taller "Interculturalidad y Derecho de los Pueblos Indígenas. Enero 2010.

\section{Sección 2. Las lenguas mesoamericanas de Nicaragua}

En esta sección vamos a recapitular brevemente algunas de las principales informaciones que se han podido establecer sobre los idiomas mesoamericanos que se hablaron en Nicaragua. Para información complementaria y más detallada sobre aspectos que abordaremos aquí sólo de manera superficial o que dejaremos de lado (por ejemplo la distribución geográfica de los grupos étnicos), el lector puede referirse a las fuentes citadas en la nota 4 arriba, y en particular a Lehmann (1920) quien, además de discutir en detalle las fuentes históricas, contiene también la mayor parte de los materiales lingüísticos que han llegado hasta nosotros sobre estos idiomas.

Chorotega-mangue. Los hablantes de chorotega, también llamado mangue, eran el grupo más numeroso en el territorio de Nicaragua a la llegada de los españoles. Aunque la evidencia que lo establece es incierta y aproximativa se supone, a partir de menciones en las crónicas, que los hallazgos arqueológicos parecen confortar que habrían llegado a Centroamérica alrededor del año 800 de nuestra era ${ }^{12}$.

La mayor parte de los materiales lingüísticos recogidos directamente de los últimos hablantes que nos han quedado, por lo demás muy fragmentarios y limitados, fueron recopilados por el investigador alemán Karl Berend en 1874. A ese fondo básico se pueden añadir las palabras que había reunido anteriormente E. Squier y las que posteriormente recogió Walter Lehmann, incluyendo nombres de lugares y otros nombres propios existentes o mencionados en las crónicas. Todas esas listas de palabras y materiales lingüísticos fueron incluidas en Lehmann 1920 donde también aparecen las referencias detalladas a las fuentes y retomadas en Quiroz (2002), como veremos adelante.

Según Lehmann (1920), pg.864, Berend fue el primero en estudiar científicamente la relación que había sido señalada originalmente por Brasseur de Beaugourgentre el chorotega-mangue y el chiapaneco, idioma que actualmente está también extinto y que se habló en la región

12 Para una revisión de la literatura antropológica relativas a los chorotegas ver Carmack (2002). 
de Chiapas en México. Si las hipótesis relativas a la llegada de los mangues a Nicaragua en el año 800 son correctas, la separación entre estos idiomas a la llegada de los españoles habría sido de unos 700 años. Lehmann incluye en su obra de 1920 (págs. 864-900) materiales sobre el chiapaneco en torno al cual hay más información que sobre el mangue.

El lingüista costarricense Juan Santiago Quiroz Rodríguez publicó en el 2002 un diccionario español-chorotega, chorotega-español, elaborado principalmente a partir de los datos reunidos por Berend y Lehmann, que incluye también un recuento e historia de las fuentes existentes y comentarios sobre la fonología y la gramática de la lengua. Para la elaboración de estos últimos, Quiroz señala haber utilizado los datos publicados sobre la gramática del Chiapaneca en Albornoz (1875) y Adams (1887), basándose en la proximidad postulada entre las dos lenguas.

El chiapaneco y el mangue han sido incluidas en la familia oto-mangue que deriva su nombre del otomí, el idioma más septentrional de la familia, todavía hablado en México, y el mangue o chorotega que es el miembro situado más al Sur. En esta familia de lenguas fue también incluido el subtiava.
Dado que la familia oto-mangue (Campell 1979) se caracteriza por una gran riqueza supra-segmental -son idiomas en los cuales los tonos juegan un rol fonológico como en el chino- es de suponer que la poca información que nos ha quedado sobre ellas es todavía más incompleta que lo que parece.

Nicarao-Nahuatl. De acuerdo a la información recopilada por los cronistas, en alguna medida confortada por los hallazgos arqueológicos y otras fuentes de información, los nicaraos habrían irrumpido en el territorio de Nicaragua alrededor del año 1200 de nuestra era, estableciéndose en la región de Rivas y otros segmentos de la franja del Pacifico a detrimento de los chorotegas.

Sobre el idioma de los nicaraos, una variedad del náhuatl, nos quedó muy poca información. Al igual que para las otras lenguas, la principal fuente es Lehmann (1920) pg. 1002-1018 que publicó y comentó los materiales existentes, provenientes de diferentes fuentes.

Existe una controversia en la literatura académica en cuanto a la relación entre los nicaraos y los pipiles, cuyo

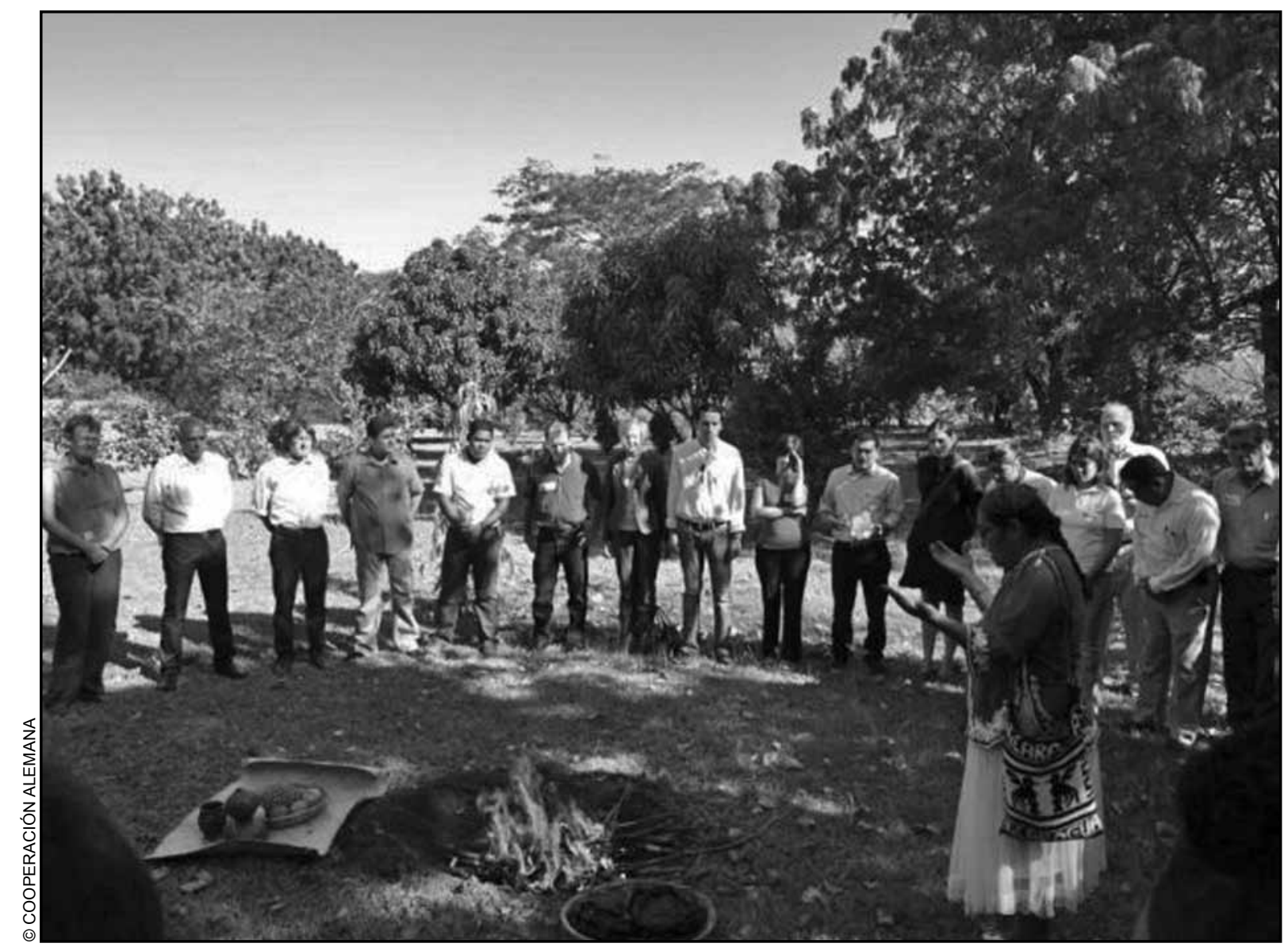

Ceremonia indígena durante el taller "Interculturalidad y Derecho de los Pueblos Indígenas. Enero 2010. 
idioma es también conocido como náhuatl o náwat, pero que se establecieron en el Salvador y Guatemala muy anteriormente (alrededor del año 600 de nuestra era $)^{13}$.

Lehmann basándose en una interpretación histórica de los materiales lingüísticos que quedaron, así como también en las fechas postuladas en las crónicas, y bajo el supuesto de que los nicaraos llegaron directamente de México a Nicaragua, postuló que el idioma de los nicarao sería más cercano a dialectos del náhuatl hablados en México que al Pipil centroamericano, ya que éste se habría separado del tronco central anteriormente, lo que también se evidenciaría en la conservación de rasgos "arcaicos".

Fowler (1989), bajo supuestos distintos en relación a la evolución histórica de las variantes del náhuatl y asumiendo una historia migratoria más compleja, que incluye desplazamientos de estos grupos al interior de Centroamérica, postula una relación más estrecha entre los pipiles y los nicaraos. La escasez de los materiales lingüísticos disponibles para el nicarao dificulta el esclarecimiento de estos temas.

El pipil es todavía hablado en el Salvador donde existe un proyecto de rescate de este idioma -ver el articulo de Jorge Lemus en este mismo número de Wani-. Por otra parte, varios dialectos del náhuatl son todavía hablados por numerosas personas en México.

Subtiava. El subtiava fue hablado por un grupo indígena que en las crónicas es denominado maribios, menos numeroso que los chorotegas y los nicaraos, y que habitaba en la zona de la cordillera del mismo nombre. Este grupo sobrevivió en el reducto indígena de Subtiava que ahora es un suburbio de León. Como ya señalamos anteriormente, la mayor parte de los materiales de los que disponemos sobre esta lengua fueron recogidos por Lehmann (1920) págs. 910-978 durante su estadía en Nicaragua en 1908-1909. Lehmann, que incluyó materiales sobre esta otra lengua en el segmento de su obra que acabamos de mencionar, fue el primero en señalar la relación estrecha entre el subtiava y el tlapaneca (también llamado yopi), varios dialectos del cual son todavía hablados en el sur de México, en el estado de Guerrero, por unas 100, 000 personas. No está claro cuándo ocurrió la llegada de los maribios o subtiava a Nicaragua, pero es probable que su venida haya sido motivada por las mismas razones que la llegada de los mangues o de los nicarao, es decir, entre el año 800 y el 1,200.

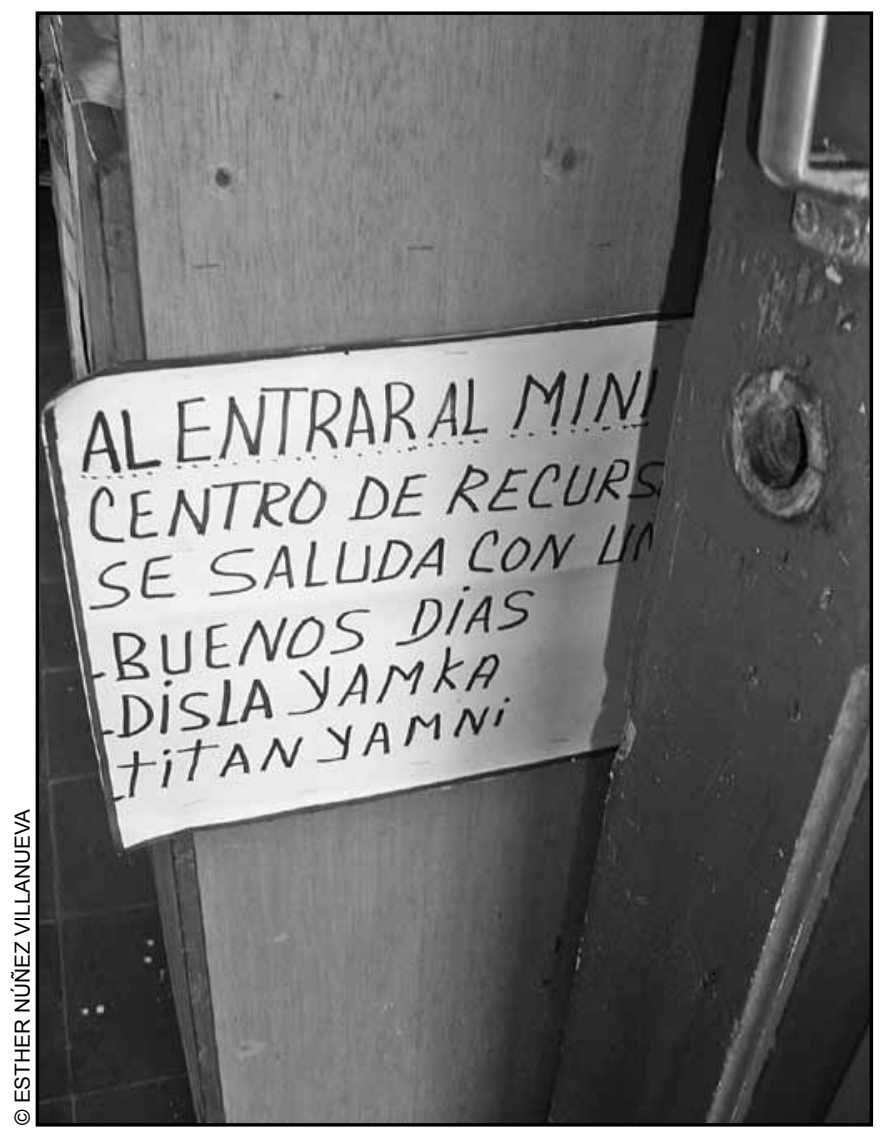

Cartel trilingüe en la escuela primaria de Karawala.

El subtiava y su pariente el tlapaneca han sido clasificados como miembros de la familia oto-mangue, es decir, que están emparentados lingüísticamente con el chorotega (o mangue). No hay evidencia segura de que el idioma haya sido hablado en otras partes de Centroamérica.

Resumiendo, entonces, de los tres idiomas mesoamericanos que tenemos noticias que se hablaron en Nicaragua solo tenemos materiales muy fragmentarios. Para dos de ellos, el nicarao y el subtiava han sobrevivido parientes cercanos en México: el nahuatl y el tlapaneca respectivamente.

En el caso del nicarao existe también todavía viva en Centroamérica una lengua afín, el pipil que se habla todavía en alguna medida en el Salvador.

En el caso del mangue o chorotega, del pariente más cercano del que se tiene noticia, el chiapaneco, se conservó un número mayor de materiales que interesa recopilar y analizar. Por otra parte conviene señalar que

13 Para una discusión sobre el tema ver la sección correspondiente al nicarao en Constenla (1994): $201-204$. 
según "Catálogo de las lenguas indígenas nacionales" publicado por el Instituto de Lenguas Indígenas de México (INALI (2008)), pg. 40-41 segunda sección, el chorotega-mangue y el subtiava constituyen un subgrupo llamado "Tlapaneco-mangueano" de la subfamilia "Oto-mangue del oeste". Sería interesante conocer los fundamentos de esta clasificación, según la cual el tlapaneco sería también el idioma sobreviviente más cercano del chorotega-mangue, y la separación temporal entre estos dos idioma similar a la que habría existido entre el subtiava y el chorotega.

Dado que no es ya posible - salvo tal vez de manera marginal a través del estudio de las toponimias- reunir más información sobre nuestros idiomas mesoamericanos, sólo nos queda profundizar el análisis de los materiales que quedaron o estudiar los idiomas parientes que han sobrevivido hasta nuestros tiempos, para poder aprender un poco más sobre por lo menos las características generales de nuestros idiomas ancestrales.

En el contexto de las luchas indígenas que motivaron plantear el examen de la cuestión de las lenguas mesoamericanas de Nicaragua, dado que el chorotega fue también hablado en Honduras y Costa Rica y que el pipil sobrevive todavía en el Salvador, nuestra investigación incluyó visitas a los países vecinos de Costa Rica, Honduras y el Salvador, para recoger información sobre la manera en que este tema se plantea allá, para informarnos sobre la tradición de estudios lingüísticos y la visión que en estos países existe sobre el tema. Sobre esto vamos a hablar en la sección siguiente.

\section{Sección 3. Visitas de investigación a los países vecinos}

Dado que las crónicas y la literatura académica nos informan que el chorotega fue hablado también en Costa Rica y Honduras, y que los náhuatl (pipiles) tuvieron importante presencia en El Salvador, nuestra investigación incluyó visitas a esos países para conocer de la historia y situación lingüística actual de los indígenas ahí, y en especial de la situación lingüística de los indígenas de cultura mesoamericana emparentados con los indígenas del Pacifico y Centro de Nicaragua.

La visita a Costa Rica fue realizada en noviembre del 2007, durante una primera fase de esta investigación. La visita a Honduras se desarrolló entre el 22 de junio y el primero de julio y la que realizamos a El Salvador ocurrió entre el 23 de noviembre y 3 de diciembre del 2009. Nuestros objetivos eran hacer contactos con lingüistas y hablantes de las lenguas, conocer de las lenguas indígenas todavía habladas y recoger materiales bibliográficos pertinentes a nuestra investigación.

3.1. Costa Rica (noviembre 2007). Durante la visita que hice a Costa Rica recibí apoyo en mi recopilación bibliográfica e investigaciones principalmente por el Dr. Juan Diego Quezada de la Universidad Nacional y el Dr. Adolfo Constenla de la Universidad de Costa Rica, estos dos lingüistas forman parte de los miembros fundadores de la Asociación Centroamericana de Lingüística (ACALING) y son especialistas en el estudio de los idiomas indígenas de Costa Rica. Ambos han orientado sus investigaciones sobre todo hacia los idiomas de la familia chibcha, de los que varios sobreviven en Costa Rica.

Adolfo Constenla es autor de una gramática del guatuso, idioma todavía vivo y es próximo al rama de Nicaragua. Es también hacia esas lenguas chibchas que se orienta la mayor parte de la investigación lingüística en Costa Ricalenguas, literatura oral y mitologías chibchas interesan particularmente en la Universidad de Costa Rica (U.C.R), que tiene un programa de estudios de lingüística con una gran trayectoria. De particular importancia son las investigaciones históricas realizadas por Adolfo Constenla y sus alumnos.

En la Universidad Nacional Autónoma (UNA) con sede en Heredia, Juan Diego Quesada y varios colaboradores y discípulos suyos han creado un centro de lingüística descriptiva de las lenguas de la Baja Centroamérica. Esto incluiría no solo los idiomas chibchas (rama de Nicaragua y paya o pech de Honduras) sino también los idiomas misumalpas, al garifuna, al tol, tolupan o jicaque, y a varios idiomas ya dados por muertos como el lenca El Salvador y Honduras o el cacaopera de El Salvador (matagalpa en Nicaragua).

Dentro de las investigaciones lingüísticas realizadas recientemente en Costa Rica destaca -en relación a nuestros propósitos - el trabajo de Juan Santiago Quiroz Rodríguez. Este lingüista que es profesor universitario en la región de Nicoya, una región que formó parte del territorio de los chorotegas y de Nicaragua y es autor de un "diccionario español-chorotega, chorotega español" publicado en 1999. También hizo su tesis de grado en la UCR sobre el chorotega.

Según mi conocimiento, Juan Santiago Quiroz es el único lingüista costarricense que trabaja sobre uno de 
nuestros idiomas mesoamericanos. Los otros dos -el nicarao y el subtiava- no se hablaron en Costa Rica, sin embargo, historiadores, arqueólogos y otros y académicos costarricenses han realizado estudios sobre la región arqueológica e histórica denominada la Gran Nicoya que incluye la franja del Pacifico de Nicaragua-ver por ejemplo el volumen 18-19 de la revista Vínculos publicada en 1994 por el Museo Nacional, o el trabajo de la historiadora Ibarra Rojas (2001) sobre la región. Durante nuestra visita a Costa Rica procuramos adquirir las obras referentes a nuestro tema de estudio publicadas en este país.

Para describir la situación actual de las lenguas indígenas de Costa Rica solicitamos a Juan Diego Quesada un artículo que estamos publicando en este mismo número de Wani.

3.2. Honduras. Para la visita a Honduras (del 22 de junio al primero de julio 2010) contaba con el apoyo de la Dra.
Gloria Lara, antropóloga de la Universidad Pedagógica, y de la lingüista Marcela Carías de la Universidad Nacional Autónoma de Honduras (UNAH). La doctora Lara es especialista en historia antigua de Honduras y en sus poblaciones indígenas actuales. La profesora Marcela Carías ha hecho estudios sobre varios de los idiomas indígenas de Honduras. Con ellas sostuve reuniones durante mi visita para informarme y orientarme en mis actividades.

Desafortunadamente, durante mi visita a Honduras la situación política se puso muy tensa -el 28 de junio 2009, mientras estaba allá tuvo lugar el golpe de estado que derrocó al gobierno de Manuel Zelaya. Por esta razón no pude viajar al interior del país ni encontrarme con personas que me pudieran dar más información sobre los así llamados "indios del Paraíso" e "indios de Santa Bárbara", que aparecen ocasionalmente mencionados en la literatura, por ejemplo, Rivas (93) pg. 47.

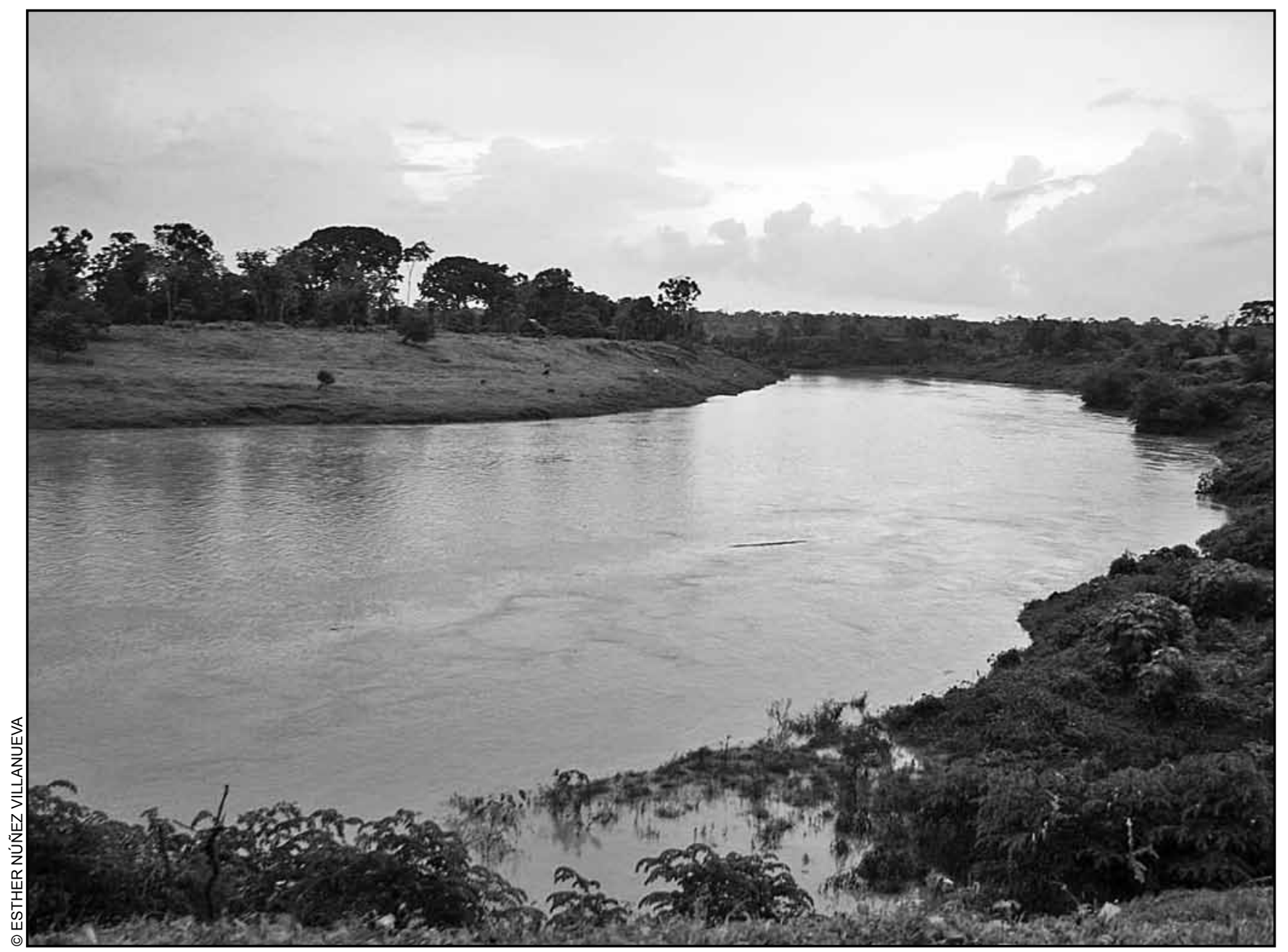

Vista del Río Grande. 2009. 


\section{La situación lingüística en Honduras}

En Honduras, además de hablantes del miskito $(50,000)$, y del sumo en la variante tawahka (500), hay unas 1000 personas que hablan todavía el idioma pech o paya, el más nórdico de los idiomas chibchas. Hay también unos 300 hablantes del idioma tol o tolupán, conocido también como jicaque, en el reducto de la Montaña de la Flor. La clasificación genealógica de este último idioma es todavía incierta.

Los garífunas, cuyo idioma pertenece a la familia arawaka, son originarios de la isla de Saint Vincent en las Antillas, y fueron desplazados por los ingleses a la isla de Roatán en Honduras en 1797, desde donde se diseminaron a otros lugares de Centroamérica, incluyendo Nicaragua y Belice. En Honduras, según el último censo del 2001, son 46,448, pero hay muchos que viven en el extranjero. No todos conservan el idioma.

En la zona fronteriza con Guatemala hay también un cierto número de chortis de nacionalidad hondureña (en el censo del 2001 se da la cifra de 34,453 personas). Usualmente han perdido el idioma, que es hablado en Guatemala.

Los negros o criollos de habla inglesa son en Honduras 12,370, según el censo del 2001. El censo del 2001 también da la cifra de 279, 507 lencas, pero el idioma ya no se habla desde hace mucho tiempo, ni en Honduras ni en el Salvador.

Hay evidencia arqueológica y documental, al igual que toponimias que indican la antigua presencia chorotega en la región de Choluteca y una tenue presencia náhuatl en el noroeste del territorio de Honduras. En lo que concierne a estos indígenas mesoamericanos que son nuestro particular objeto de interés en esta investigación, sus descendientes no aparecen reconocidos en los inventarios contemporáneos de minorías étnicas como las de Rivas (1993) o Hérranz (1996). Debido a la tensión política, como ya señalé, no pude averiguar si los "indios del Paraíso" o los "indios de Santa Bárbara" mencionados arriba representan una tradición reivindicativa de descendientes de estos grupos étnico-lingüísticos; un tema que quedó pendiente en esta investigación.

En los últimos años se han dado iniciativas en Honduras tendientes a integrar a los programas escolares el estudio de las lenguas y las culturas indígenas. Esto se ha dado tanto para las lenguas que todavía se hablan, a través de programas de educación bilingüe; como las que ya no se hablan, a través de programas de revitalización lingüística, ha sido el caso del chortí que todavía se habla en Guatemala, o de revitalización cultural como se ha venido proponiendo para los lencas.

3.3. El Salvador. Mi visita a El Salvador ocurrió entre el 23 d noviembre y el 3 de diciembre del 2009. Para ella conté con el apoyo y guía del Dr. Jorge Lemus, lingüista y director de investigaciones de la Universidad Don Bosco. El Dr. Lemus es el responsable de un programa de revitalización del idioma náwat o pipil que se desarrolla desde hace varios años a partir de comunidades indígenas de la región de Sonsonate y se ha extendido a algunas escuelas fuera de este departamento.

En el Salvador se hablaban al inicio de la colonia varios idiomas indígenas, los principales eran: el lenca, en una variedad diferente de la de Honduras, el cacaopera, próximo del matagalpa, y el nawat. Actualmente el lenca y el cacaopera están extintos y de la variedad de náhuatl hablada en el Salvador llamado "nawat" sólo sobreviven unos 200 hablantes y semi-hablantes principalmente radicados en los alrededores de Sonsonate, sobre todo en el pueblo de Santo Domingo de Guzmán (de nombre indígena Witzapan). Es a partir de esa región, que el proyecto de revitalización y enseñanza del idioma nawat en las escuelas se ha venido expandiendo.

Fui invitado a participar en el Séptimo Seminario Lingüístico-Metodológico para profesores de nawat que tuvo lugar del 24 al 27 de noviembre en Sonsonate. En este programa, que tiene seis años de existir, 28 profesores enseñan actualmente la lengua nawat como segundo idioma a niños indígenas. Se atendía a 3.000 niños en el 2009 y para el año escolar siguiente se espera atender a 3,500. Desde el punto de vista de nuestra investigación sobre los idiomas mesoamericanos de Nicaragua, el programa de revitalización del nawat de El Salvador es de gran interés.

En este mismo número de Wani estamos publicando dos artículos de Jorge Lemus: uno sobre la identidad y las reivindicaciones indígenas en el Salvador, y otro a propósito de este programa de revitalización del nawat, que a inicios del 2010 acaba de recibir el respaldo oficial del estado. A ellos referimos al lector para mayores detalles.

Además de la adquisición de materiales bibliográficos e información, la visita a El Salvador nos permitió hacer contactos con hablantes y maestros del nawat que están dispuestos a venir a Nicaragua para compartir sus experiencias y conocimientos con los indígenas nicaragüenses, en particular con los que son descendientes 
de hablantes del Nahuatl. Para estas colaboraciones futuras contamos con el apoyo del Dr. Jorge Lemus, miembro fundador de la Asociación Centroamericana de Lingüística (ACALING).

\section{Sección 4. Resultados y Perspectivas}

Nuestro trabajo investigativo sobre los idiomas mesoamericanos de Nicaragua tenía como principal objetivo apoyar y ayudar a fundamentar el conocimiento de sus raíces culturales por parte de las agrupaciones indígenas que se reclaman descendientes de los grupos étnicos que poblaban la región del Pacifico y Centro del país a la llegada de los españoles. Desde esa perspectiva, nuestra ambición era principalmente inventariar los materiales bibliográficos, investigaciones e iniciativas existentes sobre las lenguas y culturas en cuestión tanto en Nicaragua como en los países vecinos. Para nuestro trabajo en el extranjero solicitamos el apoyo de académicos de cada país contribuyendo de esta forma a conformar una red lingüistas que colaboren en el estudio y promoción del patrimonio lingüístico que compartimos.
A continuación presentaremos una lista de los principales resultados de esta investigación, así como de las perspectivas de aprovechar y ampliar el trabajo de investigación y colaboración que hemos iniciado.

4.1. La creación de un fondo bibliográfico. La creación del fondo bibliográfico que hemos venido constituyendo y que estamos explotando por primera vez en esta publicación, tiene el objetivo de alimentar actividades educativas y divulgativas por parte de las organizaciones indígenas y a la vez contribuir a crear condiciones para poder desarrollar investigaciones sobre las lenguas y culturas mesoamericanas de Nicaragua

Aparte de las crónicas de la colonia y los clásicos de la literatura académica sobre las poblaciones indígenas de la región que hemos podido conseguir en las librerías centroamericanas, los libros que hemos estado reuniendo son principalmente obras contemporáneas sobre las lenguas y culturas indígenas, la historia antigua, y la literatura nacional identitaria publicadas en cada uno de los países que hemos visitado: Costa Rica, El Salvador, Honduras.

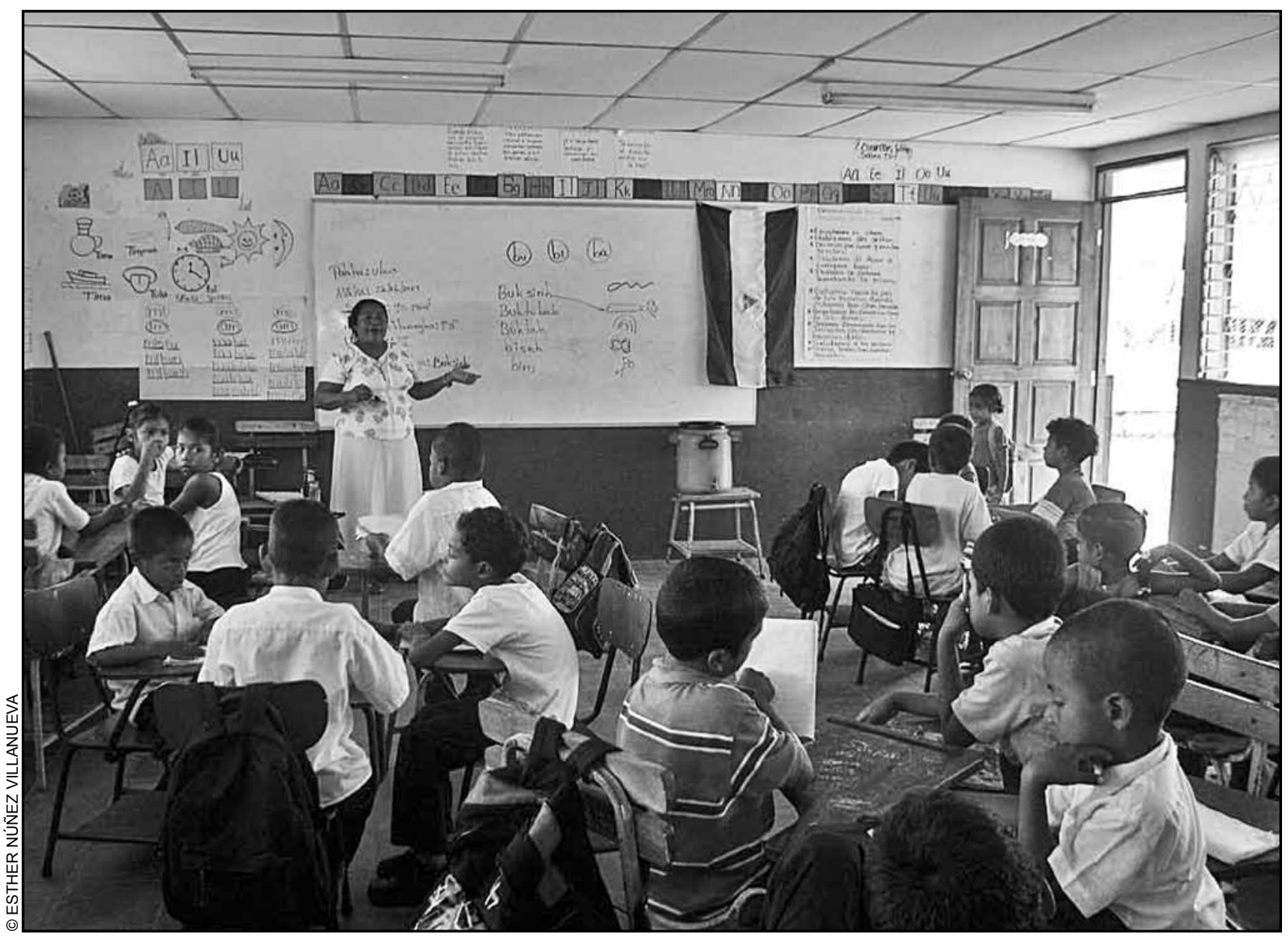

Clase en idioma ulwa. Karawala, 2009. 
El fondo documental ambiciona reunir en particular todos los materiales que quedaron sobre las lenguas así como los estudios realizados sobre ellas en cada uno de los países donde fueron habladas.

Naturalmente, hasta ahora hemos juntado solo una parte pequeña de un conjunto mucho más amplio de publicaciones de primera importancia sobre nuestros indígenas mesoamericanos. Convendría en particular alimentar el fondo con obras contemporáneas de naturaleza académica (historia, antropología, arqueología, geografía, lingüística...etc.), publicadas tanto en español como en lenguas extranjeras fuera del ámbito centroamericano.

La colección de libros que conseguimos reunir podría constituirse en un fondo y punto de partida para una biblioteca especializada que podría servir también para sustentar cursos universitarios sobre nuestros indígenas mesoamericanos. A este respecto conviene señalar que en la reunión que sostuvimos en la comunidad de San Lucas con dirigentes de la coordinara chorotega el 16 de septiembre del 2009, varias de las sugerencias presentadas por los dirigentes iban en la dirección de crear un centro de estudios permanente sobre la cuestión indígena en el lado hispanohablante del país.

Por el momento, la colección de libros adquirida con los fondos del proyecto está en poder de la revista Wani, pero lo ideal sería que estuviera localizado en un lugar accesible a las organizaciones indígenas concernidas.

\subsection{Creación de redes de colaboración a nivel} centroamericano. El trabajo realizado en el marco de este proyecto, desde que se inició con una visita a Costa Rica en el 2007, contribuyó a la creación en el mes de agosto del 2009 de la Asociación Centroamericana de Lingüística (ACALING). Esta asociación nació bajo el patrocinio de la Universidad Nacional de Costa Rica (UNA) en ocasión de un simposio de lingüística organizado en Heredia por la escuela de literatura y ciencias del lenguaje de esa universidad.

El objetivo de esta red de colaboración es facilitar y potenciar las investigaciones y otras actividades relativas a las lenguas indígenas -como proyectos de revitalización, simposios, actividades educativas...etc- a través de las fronteras centroamericanas. También se promoverán los contactos entre las poblaciones del mismo origen lingüístico y cultural a través de las fronteras; porque los espacios nacionales tienden a establecer separaciones artificiales entre las poblaciones indígenas, como ocurre por ejemplo entre los miskitos y mayangnas de Honduras y Nicaragua.

Hacia esas metas hemos estado trabajando a lo largo de la investigación que estamos aquí resumiendo, particularmente durante nuestras visitas a los otros países centroamericanos.

Un ejemplo de relaciones de este tipo que estamos queriendo propiciar son los contactos entre miembros del programa de revitalización de la lengua náwat del Salvador (ver el artículo de Jorge Lemus en este mismo número) y las organizaciones indígenas pertinentes en Nicaragua.

\subsection{Divulgación y Profundización de los conocimientos} y de las actividades en curso sobre nuestros idiomas indígenas mesoamericanos. Nuestra revista Wani, que ha servido de vehículo para hacer conocer las investigaciones sobre las lenguas indígenas de la Costa Atlántica, está dedicando este número a las lenguas indígenas del Pacifico y Centro de Nicaragua. Esperamos poder publicar en futuras ediciones de nuestra revista trabajos que profundicen los elementos básicos de información contenidos en este número, particularmente aquellos que puedan ser añadidos al acervo de conocimientos sobre las lenguas.

A ese último propósito podemos señalar, dentro de las sugerencias emitidas por los líderes indígenas de las coordinadora chorotega con quienes nos entrevistamos, la posibilidad de reunir términos léxicos de origen indígena referentes a objetos así como actividades tradicionales y términos geográficos de los pueblos y comunidades que no hayan sido hasta ahora incluidos en las listas de términos heredados de las culturas ancestrales y que forman parte de la tradición oral de estos pueblos.

Estamos incluyendo en este número un artículo de Jorge Lemus sobre el proyecto de revitalización del náwat en El Salvador y una evaluación de la situación de las lenguas indígenas en Costa Rica por el lingüista costarricense Juan Diego Quezada. Estaremos abiertos a recibir colaboraciones de parte de los lingüistas centroamericanos que estudian los idiomas mesoamericanos que compartimos.

Más allá de la frontera de Centroamérica, nos gustaría publicar información sobre las variantes del náhuatl habladas en México y sobre el idioma tlapaneca, que todavía es hablado en México y que como mencionamos en la sección 2 es el pariente más cercano de nuestros idiomas chorotega y subtiava que sobrevive. 


\section{Referencias}

Adam, Lucien (1887) La Langue chiapaneque. Viena 1887, 117 pg. -según Lehamann (1920) pg. 867, elaborado principalmente a partir de Albornoz (1875) y otros manuscritos de Brasseur de Beaubourg-.

Albornoz, Fray Juan de (1875). Arte de la lengua chiapaneca (original de mediados del siglo XVII). Paris: Ernest Léroun, éditeur.

Campbell, L. (1975) “Subtiava 1974”. International Journal of American Linguistics 41.1. 80-84.

(1979).’Middle American Languages”. En: Campbell, L. y Mithun, M. (ed.) (1979). The Languages of Native America: Historical and Comparative Assessment. (Austin: University of Texas Press).

(1985). The Pipil Language of El Salvador. Berlin: Mouton.

Carmack, Robert M. (2002) “Historia prehispánica de los chorotegas de Nicaragua. Una síntesis antropológica” Revista de Historia no. 14: 13-25, Publicación del IHNCA. Universidad Centroamericana. Managua.

Coehlo, Ruy Galvao de Andrade (1995) - segunda edición en español, original de 1955- Los negros caribes de Honduras. Tegucigalpa. Editorial Guyamuras.

Constenla Umaña, Adolfo (1987) "Elementos de fonología comparada de las lenguas misumalpas" Revista de Filología y Linguística XIII (1) (129-161). Universidad de Costa Rica.

(1991) Las Lenguas del Area Intermedia: introducción al estudio areal. Editorial de la Universidad de Costa Rica, San José.

(1994) “Las lenguas de la Gran Nicoya”. Vinculos -Revista de Antropología del Museo Nacional de Costa RicaVolumen 18-19: 191-208.

(2005) ¿Existe relación genealógica entre las lenguas misumalpas y las chibchences? “Estudios de Lingüistica Chibcha. Tomo XXIV. Universidad de Costa Rica.

(2002): “Acerca de la relación genealoógica de las lenguas Lencas y las lenguas Misumalpas” Revista de Filología y Lingüística XXVIII (1) -189-205. Universidad de Costa Rica.

Fowler, W.R.Jr. (1989) The Pipil Nicarao of Central America. Norman: University of Oklahoma Press.

Greenberg, J. (1956). "General classification of Central and South American languages”. Men and Cultures: 5th International Congress of Anthropological and Ethnological Sciences, 791-94. Philadelphia.

Hasemann, George (1991). La Etnología y la Lingüística en Honduras, una mirada retrospectiva. Estudios Antropológicos e Históricos, no. 9. Instituto Hondureño de Antropología e Historia. Tegucigalpa.

Herranz, Atanasio (1996). Estado Sociedad y Lenguaje. La política lingüística de Honduras. Tegucigalpa: Editorial Guaymuras.

Ibarra Rojas, Eugenia (2001). Fronteras étnicas en la conquista de Nicaragua y Nicoya. San José, Editorial de la Universidad de Costa Rica.

INALI (Instituto Nacional de lenguas indígenas) (2008). Catálogo de las Lenguas Indígenas Nacionales: Variantes Lingüisticas de México con sus autodenominaciones y referencias geoestadísticas. http://www.inali.gob.mx/catalogo2007/ y Diario Oficial14 de enero del 2008.

Incer Barquero, Jaime (1998). “Ubicación de los grupos indígenas de Nicaragua en los siglos XVI y XVII”. En Lothrop y otros (1998). 
Kinloch Tijerino, Frances (2006). Historia de Nicaragua, Segunda edición. Instituto de Historia de Nicaragua y Universidad Centroamericana. Managua.

Lange, F.W., Payson D.Sheets, Aníbal Martinez y Suzanne Abel-Vidor (1992). The Archeology of Pacific Nicaragua. University of New México Press, Albuquerque.

Lehmann, W (1910) “Ergebnisse einer Forschungreise in Mittelamerika und México 19071909”. En: Zeitschtift fur Ethnologie, Jahrg. 1910, Heft 5, pp. 687749.

(1920): ZentralAmerika, Teil I, Die Sprache ZentralAmerika in ihren Beziehungen zueinander sowie zu SudAmerika und Mexiko. Berlin, Verlag Dietrich Reimer.

Lothrop, Samuel Kirkland (y otros autores) (1998) Culturas Indígenas de Nicaragua, tomo 1. Managua, Hispamer (segunda edición en el 2004).

(1998) Las culturas indígenas prehispánicas en Nicaragua y Costa Rica. En Lothrop y otros (1998).

Olguín Martinez, Gabriela -editora- (2006). Estudio de base sobre las condiciones de vida de los pueblos indígenas del Pacífico, Norte y Centro de Nicaragua. San José, Oficina Internacional del Trabajo, 2006.

Quesada, Juan Diego (2007). The Chibchan Languages. Cartago. Editorial Tecnológica de Costa Rica.

Quiroz Rodriguez, Juan Santiago (2002) Diccionario Español-Chorotega, Chorotega-Español. Editorial de la Universidad de Costa Rica, San Jose. (Contiene también un esbozo gramatical)

Rivas, Ramón (1993). Pueblos Indígenas y Garifuna de Honduras (una caracterización). Tegucigalpa: Editorial Guaymuras.

Rizo, Mario (2008). Historia del Pueblo Indígena de Telpaneca. Publicado con el patrocinio de la UNICEF, Managua. Mario Rizo.

Romero Vargas, German (editor) (1992)a. Persistencia Indigena en la Nicaragua de Hoy. Managua, CIDCA-UCA.

-------- (1992)b. “Las poblaciones Indigenas de Nicaragua, 1492-1821”. En Romero Vargas (editor) (1992).

(2003). Historia de Nicaragua. Hispamer, Managua.

Salamanca, Danilo (1993). "Los idiomas indígenas y de la población negra de Nicaragua". América Indígena. Vol LIII, no. 1-2: $23-41$

(1994). "Los idiomas indígenas del Pacífico". Wani no. 15, Marzo de 1994.

(2007) “El trabajo de los lingüistas en CIDCA y Wani”. Wani no.51. Octubre-Diciembre 2007.

Schultze Jena, Leonhad (1982). Gramatica Pipil y Diccionario Analítico. Ediciones Cuscatlan. San Salvador.

Squier, Ephraim George (1970) (publicado por primera vez en inglés en 1852) Nicaragua, sus gentes y paisajes. Editorial Universitaria Centroamericana. San José. 